OPEN ACCESS

Edited by:

Ishu Saraogi,

Indian Institute of Science Education

and Research, India

Reviewed by:

Heinz Neumann,

Darmstadt University of Applied

Sciences, Germany

Weimin Xuan,

Nankai University, China

*Correspondence:

Ryan A. Mehl

Ryan.Meh/@oregonstate.edu

Specialty section: This article was submitted to

Chemical Biology,

a section of the journal

Frontiers in Chemistry

Received: 14 December 2021

Accepted: 25 January 2022

Published: 21 February 2022

Citation:

Van Fossen EM, Grutzius S, Ruby CE, Mourich DV, Cebra C, Bracha S, Karplus PA, Cooley RB and Mehl RA (2022) Creating a Selective Nanobody Against 3-Nitrotyrosine

Containing Proteins.

Front. Chem. 10:835229.

doi: 10.3389/fchem.2022.835229

\section{Creating a Selective Nanobody Against 3-Nitrotyrosine Containing Proteins}

\author{
Elise M. Van Fossen ${ }^{1}$, Sonia Grutzius ${ }^{1}$, Carl E. Ruby ${ }^{2}$, Dan V. Mourich ${ }^{2}$, Chris Cebra ${ }^{2}$, \\ Shay Bracha ${ }^{3}$, P. Andrew Karplus ${ }^{1}$, Richard B. Cooley ${ }^{1}$ and Ryan A. Mehl ${ }^{1 *}$ \\ ${ }^{1}$ Oregon State University, Department of Biochemistry and Biophysics, Agricultural and Life Sciences, Corvallis, OR, \\ United States, ${ }^{2}$ Oregon State University, Department of Clinical Sciences, College of Veterinary Medicine, Corvallis, OR, \\ United States, ${ }^{3}$ Department of Small Animal Clinical Sciences (VSCS), Texas A\&M College of Veterinary Medicine and Biomedical \\ Sciences, College Station, TX, United States
}

A critical step in developing therapeutics for oxidative stress-related pathologies is the ability to determine which specific modified protein species are innocuous by-products of pathology and which are causative agents. To achieve this goal, technologies are needed that can identify, characterize and quantify oxidative post translational modifications (oxPTMs). Nanobodies (Nbs) represent exquisite tools for intracellular tracking of molecules due to their small size, stability and engineerability. Here, we demonstrate that it is possible to develop a selective $\mathrm{Nb}$ against an OxPTM protein, with the key advance being the use of genetic code expansion (GCE) to provide an efficient source of the large quantities of high-quality, homogenous and site-specific oxPTM-containing protein needed for the $\mathrm{Nb}$ selection process. In this proof-of-concept study, we produce a Nb selective for a 3-nitrotyrosine (nitroTyr) modified form of the 14-3-3 signaling protein with a lesser recognition of nitroTyr in other protein contexts. This advance opens the door to the GCE-facilitated development of other anti-PTM Nbs.

Keywords: genetic code expansion, nanobody, oxidative post translational modification, nitrotyrosine, single domain antibody

\section{INTRODUCTION}

Oxidative post-translational modifications (oxPTMs) are formed by small molecule oxidants reacting with proteins under both normal and oxidative stress conditions and their heterogeneity in terms of the types, locations and the extents of the modification make it challenging to pin-point the effects they exert. Regardless, the study of oxPTMs remains critical as these modifications are often identified in disease pathology and show promise as disease prediction tools (Tomin et al., 2019).

The oxPTM 3-nitrotyrosine (nitroTyr, nY) is produced by the formation of peroxynitrite-derived radicals and their subsequent reaction with tyrosine side chains (Souza et al., 2008). Its accumulation in over 100 distinct proteins is associated with numerous disease pathologies (Pacher et al., 2007). NitroTyr-modified proteins have been shown to cause functional changes that can contribute to disease (Franco et al., 2013; Bartesaghi and Radi 2018; Ferrer-Sueta et al., 2018; Radi, 2018; Porter et al., 2020), but this has been possible in only a few cases, as it is challenging to define which specific nitroTyr protein species are innocuous by-products of pathology and which are causative agents. As such knowledge is required for the development of effective therapeutics for oxidative stress-related pathologies, there is a critical need for tools to identify, characterize and quantify nitroTyr-modified proteins. 
One such tool has been anti-nitroTyr antibodies (nitroTyrAbs), which have enabled immune-localization of nitroTyr proteins (Moller et al., 2019) and nitroTyr-specific proteomic profiling (Herce-Pagliai et al., 1998). Most commonly employed is a nitroTyr-Ab specific to the PTM itself (Beckmann et al., 1994), though protein and site-specific nitroTyr-Abs have also been developed (Khan et al., 2017). Despite the utility of nitroTyrAbs for the identification and enrichment of nitrated proteins, their large size has prevented their use for tracking proteins in live cells, which would allow nitroTyr-induced changes in client binding and subcellular location to be monitored.

Recently, nanobodies (Nbs)-single-domain antibody fragments derived from the heavy-chain of immunoglobulins of camelids and much smaller than Abs ( $15 \mathrm{kDa}$ vs. $-150 \mathrm{kDa})-$ have been shown to be effective for the intracellular tracking of proteins (Moeglin et al., 2021). Additionally, Nbs are well suited for binding epitopes that are inaccessible to traditional Abs due to their protruding, convex paratopes (Muyldermans 2013; Pardon et al., 2014). The ability to generate nitroTyr-protein specific Nbs would allow the full suite of $\mathrm{Nb}$ capabilities to be harnessed for both in vitro and in cell work, including for instance, the modulation of protein activity by targeting nitroTyr proteins with a covalent nanobody (or "GlueBody") for degradation in order to observe the downstream effects on redox signaling (Bery et al., 2019; Cheloha et al., 2020; de Beer and Giepmans 2020; Zhang et al., 2021).

$\mathrm{Nb}$ selections require high-quality, homogenous target protein (Pardon et al., 2014), and the inability to make such protein has been a general barrier to creating Nbs against oxPTMs. Traditionally, oxPTMs are chemically introduced into proteins but this process generates multiple types of chemical modifications with minimal ability to control site-specificity (Ischiropoulos et al., 1992; Neumann et al., 2008; Gerding et al., 2019). Indeed, to our knowledge, no $\mathrm{Nb}$ has yet been developed against any oxPTM and only a single example has been published for any type of PTM (Moeglin et al., 2021). An avenue to bypass this barrier for PTMs is genetic code expansion (GCE), which can install a variety of PTMs into proteins to generate large quantities of homogenous, site-specific PTM-containing proteins (Neumann et al., 2008; Franco et al., 2013; Cooley et al., 2014a; Cooley et al., 2014b; DiDonato et al., 2014; Franco et al., 2015; Porter and Mehl 2018; Porter et al., 2019; Randall et al., 2019; Beyer et al., 2020; Jang et al., 2020; Porter et al., 2020). In GCE, non-canonical amino acids (ncAAs) are site-specifically incorporated into stop codons via orthogonal amino-acyl

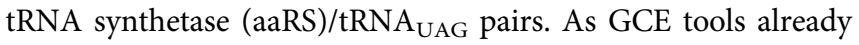
exist to incorporate nitroTyr, we sought here to use GCEproduced nitroTyr-modified proteins as proof of concept to show how Nbs can be developed against oxPTM targets.

As trial nitrated targets, we selected two hub proteins involved in cell signaling pathways: 14-3-3 (Pennington et al., 2018) and calmodulin [CaM; (Smallwood et al., 2003)]. Both govern critical processes in biology and contain multiple biologically relevant sites of tyrosine nitration (Ghesquiere et al., 2009; Nuriel et al., 2015; Zhao et al., 2017; Porter et al., 2020). After generating a nitroTyr-Nb library from an immunized camelid, we performed selections with the most promising candidate protein target and obtained a single $\mathrm{Nb}$ with a reasonable level of selectivity for certain nitrated protein targets, even in highly proteinaceous solutions. Furthermore, as a step toward developing covalent $\mathrm{Nb}$ binders, we incorporate a photocrosslinking ncAA at multiple positions in this anti-nitroTyr Nb. Though these positions did not result in high yielding crosslinking, we showcase the versatility of GCE-technology in $\mathrm{Nb}$ development, from $\mathrm{Nb}$-selections to $\mathrm{Nb}$ engineering.

\section{MATERIALS AND METHODS}

\section{Non-Canonical Amino Acids}

3-nitro-tyrosine (nitroTyr, nY) and 4-azido-phenylalanine (azidoPhe, pAzF) were purchased from Alfa Aesar (Cat. no. A11018) and Chem impex (Cat. no. 06162). NcAA solutions were prepared fresh before each use by suspending the amino acids in water and solubilizing with 1-2 $\mathrm{M}$ equivalents of $\mathrm{NaOH}$ for a final concentration of $100 \mathrm{mM}$ ncAA.

\section{Molecular Cloning}

Molecular cloning of plasmids used in this study (Supplementary Table S1) were constructed in the following manner. Previously published sequences containing at least $25 \mathrm{bp}$ of homology at their flanking ends to either the vector backbone or other fragments, were optimized for expression in E. coli and synthesized by Integrated DNA Technologies (Coralville, IA, USA, Supplementary Table S2). Gene fragments, as well as fragments containing amber stop codons were amplified with primers listed in Supplementary Table S3 using touchdown PCR (Green and Sambrook 2018) and the resulting products were separated on $0.8-1.2 \%(\mathrm{w} / \mathrm{v})$ agarose gels and purified using Gene Jet Gel Extraction Kit (ThermoFisher Scientific, Waltham, MA, United States) according to the manufacturer's instructions. Vector backbones were prepared through restriction digestion and purified through gel extraction. Fragments and vector backbones were then ligated using the SLiCE cloning protocol (Zhang et al., 2012) transformed into chemically-competent DH10B E. coli cells and selected on LB agar plates containing the appropriate antibiotic. Colonies were selected and propagated prior to purification. Genetic sequences of each plasmid were confirmed using Sanger sequencing. The primers and template used for PCR amplification, as well as the vector backbone and restriction enzymes used for linearization are summarized for each plasmid in Supplementary Table S1. Plasmids not prepared in this study were prepared previously (see references in Supplementary Table S1).

\section{Protein Expression}

For the expression of nitroTyr and azidoPhe proteins, each TAG site containing expression plasmid was co-transformed with a GCE-machinery plasmid (see Supplementary Table S1) into BL21ai cells. All pDule2 machinery plasmids contain a p15a origin of replication and constitutively express the indicated amber suppressing Methanocaldococcus jannaschii ( $\mathrm{Mj})$ aminoacyl tRNA synthetase (aaRS)/tRNA $\mathrm{CUA}_{\mathrm{C}}$ pair. Cultures $(5 \mathrm{ml})$ were inoculated by scraping a swath of cells containing 
the appropriate plasmids from a fresh LB agar plate and were grown overnight in 2XYT (no more than $16 \mathrm{~h}$ ) in the presence of appropriate antibiotics. Overnight cultures were then used to inoculate expression cultures of ZY-AIM media [0.1-1.0 L, (Studier 2005)], supplemented with appropriate antibiotics. For the expression of nitroTyr and azidoPhe proteins, the media was also supplemented with nitroTyr and azidoPhe (at final concentration of $1 \mathrm{mM}$, diluted from a freshly prepared $100 \mathrm{mM}$ solution as described above). Cultures were grown with constant shaking at $275 \mathrm{rpm}$ in baffled flasks at $37^{\circ} \mathrm{C}$ until they reached an $\mathrm{OD}_{600}$ of 1.5 , wherein they were induced with a final concentration of $0.1 \%$ arabinose. Upon induction, the temperature was decreased to $25^{\circ} \mathrm{C}$ and cultures expressed for an additional $30 \mathrm{~h}$ prior to cell harvesting.

\section{Protein Purification}

Cell pellets were resuspended in a lysis/wash buffer (50 mM Tris, $500 \mathrm{mM} \mathrm{NaCl}, 5 \mathrm{mM}$ imidazole, $\mathrm{pH}$ 7.5) and lysed using a M110P microfluidizer system (Microfluidics Corp, Newton, MA, United States) set at 18,000 psi. Cell debris was pelleted at 20,000 rcf for $25 \mathrm{~min}$ at $4^{\circ} \mathrm{C}$ and clarified cell lysate was recovered. To bind $\mathrm{His}_{6}$-tagged protein, clarified cell lysate was then incubated with TALON resin (Takara Bio, Japan) at $4^{\circ} \mathrm{C}$ for $1 \mathrm{~h}$ with rocking. Resin was collected and extensively washed with 50 resin bed volumes (bv) of lysis buffer. At this point, for proteins containing an N-terminal His- $b d$ NEDD8 tag (Frey and Gorlich 2014), the protein-bound resin was resuspended in 3 bvs of storage buffer ( $50 \mathrm{mM}$ Tris, $500 \mathrm{mM} \mathrm{NaCl}, 10 \%$ glycerol). Histag free $b d \mathrm{NEDP} 1$ (a kind gift from Dirk Gorlich, Addgene $\# 104129,100 \mathrm{nM}$ ) was added to the resuspension and the solution was incubated for $1.5 \mathrm{~h}$ at room temperature with rocking. The resin was retained in a column and cleaved nanobody was collected in the flow-through.

For all other constructs, bound protein was eluted from the TALON resin by incubation with five bv's of Elution Buffer (lysis buffer with $200 \mathrm{mM}$ imidazole) and desalted into storage buffer using a PD-10 desalting column (GE Health Sciences). When cleavage of a $\mathrm{His}_{6}$-SUMO tag was required, Ubiquitin-Like Protease 1 (ULP1) was added to the desalted solution and was incubated overnight at $4^{\circ} \mathrm{C}$. Following incubation, cleaved protein was re-flowed over TALON resin to bind the cleaved tag, and the flow-through containing the $\mathrm{Nb}$ was collected.

$\mathrm{Nb}-\mathrm{G} 5$ was further purified by size exclusion chromatography using a Superdex $20010 / 300$ column in storage buffer. When necessary, the protein solution was concentrated by using a $3 \mathrm{kDa}$ MWCO Vivaspin spin-concentration filter (GE Health Sciences). Protein concentration was determined by absorbance at $280 \mathrm{~nm}$ using primary sequence calculated extinction coefficients, flashfrozen in liquid nitrogen and stored at $-80^{\circ} \mathrm{C}$.

\section{Mass Spectrometry of Nitrated Proteins}

Purified protein was exchanged into LC-MS grade water or $50 \mathrm{mM}$ tri-ethyl-ammonium bicarbonate with PD-10 columns (Cytiva) diluted to $50 \mu \mathrm{M}$ and analyzed with the Waters Synapt G2 Mass Spectrometer at the Mass Spectrometry Facility at Oregon State University. The deconvoluted masses were obtained by using Waters MassLynx MaxEnt1 software.

\section{Expression and Purification of Biotinylated Proteins for Biolayer Interferometry}

Biotinylated 14-3-3 proteins were expressed in BL21ai by cotransforming pBAD-AVI-14-3-3-His plasmids (Supplementary Table S1) with the GCE machinery plasmid pDule2-3-nitroTyrA7 (Addgene \#174079) and the pEVF-GST-BirA plasmid. This plasmid expresses the fusion protein GST-BirA in a vector compatible with GCE machinery plasmids and most common expression plasmids (RSF origin, $\mathrm{CmR}$ antibiotic resistance). pBAD-AVI-eGFP-His was also co-transformed with pEVFGST-BirA to generate biotinylated eGFP as a control for Biolayer Interferometry (BLI) experiments.

Expression of biotinylated 14-3-3ß proceeded as described above except with the addition of $50 \mu \mathrm{M}$ biotin and $25 \mu \mathrm{g} / \mu \mathrm{L}$ chloramphenicol to expression cultures, and at an OD of 1.5, $0.1 \mathrm{mM}$ IPTG was added to induce the expression of GST-BirA. The purification of biotinylated $14-3-3 ß$ was identical to nonbiotinylated 14-3-3ß and biotinylation of $\mathrm{Nb}$ targets was validated by streptavidin motility-shift assay (Fairhead and Howarth 2015).

\section{Peptide Design for Library Generation}

Peptides were designed to encompass nitrated portions of calmodulin (nY99 and nY138) and 14-3-3 (nY133). The peptide for 14-3-3- nY133 BMHI shared a sequence identity of $61.9 \%$ with $14-3-3$ nitroTyr human isoform b. A peptide for general nitration was also designed, containing multiple nitroTyr flanked by $\beta$-sheets. Peptides were designed by following the described parameters for $\mathrm{Nb}$ generation against a folded protein (Trier et al., 2012) and were submitted to B-epitope predictors (http://crdd.osdd.net/raghava/abcpred/) to determine epitope effectiveness. The peptides contained a N-terminal cysteine to facilitate keyhole limpet hemocyanin $(\mathrm{KLH})$ conjugation. The peptides were synthesized by Genscript, resuspended in TBS and conjugated to KLH with the Thermo Scientific Imject MaleimideActivated mcKLH Spin Kit by following the manufacturer's instructions.

\section{Nitration of KLH}

$\mathrm{KLH}$ was nitrated by following a previously described method (Ischiropoulos et al., 1992; Beckmann et al., 1994). Briefly, KLH $(100 \mu \mathrm{g})$ was diluted into $100 \mathrm{mM}$ potassium phosphate $\mathrm{pH} 7.4$ to a final concentration of $0.160 \mu \mathrm{g} / \mu \mathrm{L}$. Peroxynitrite $(160 \mathrm{mM}$ stock concentration) was added to the solution to achieve a final concentration of $2 \mathrm{mM}$. The solution was incubated at room temperature for $14 \mathrm{~h}$, after which nitrated $\mathrm{KLH}$ was exchanged into PBS by overnight dialysis at $4^{\circ} \mathrm{C}$ (MWCO 3,000 Da). Nitration was confirmed by Western Blotting using Millipore's Polyclonal anti-nitroTyr Ab (Cat \# 06-284, see below section "Western Blots").

\section{Construction of $\mathrm{Nb}$ Display Libraries}

\section{Immunization of Animal}

A healthy Oregon State University owned alpaca was the host for $\mathrm{Nb}$ production. The animal was immunized over a series of six injections with a total of $700 \mu \mathrm{g}$ of a mixture of the nitrated peptides conjugated to KLH in IX PBS mixed with equal volume 
( $1 \mathrm{ml}: 1 \mathrm{ml}$ ) Sigma adjuvant System. The peptides were injected subcutaneously over the right caudal neck. Pre- and post-immune serum samples were collected. Immunizations were followed by a production bleed not exceeding $200 \mathrm{ml}$ and whole blood collected into heparinized tubes. All animal protocols were approved by the Oregon State University Institutional Animal Care and Use Committee (IACUC).

\section{Serum Analysis}

Pre- and post-immune serum samples were collected and screened for the presence of Nbs binding nitrated targets. 143-3 (WT and nY133), CaM (WT, nY99 and nY138) (5 $\mu \mathrm{g})$, and HCT116 cell lysate as a positive control were separated on $15 \%$ SDS-PAGE gels, transferred to PVDF membrane, blocked with $5 \%(\mathrm{w} / \mathrm{v})$ nonfat milk in TBST and probed with either pre- or post-immunization serum, rocking for $16 \mathrm{~h}$ at room temperature. After rinsing three times with TBST, the membranes were than incubated with anti-llama horseradish peroxidase (HRP) conjugated secondary antibody (goat anti-llama IgG HRP, ab112786, Abcam, Cambridge, United Kingdom) diluted 1: 20,000 in $5 \%$ nonfat milk/TBST for $1 \mathrm{~h}$ at room temperature. The membranes were washed three times with TBST and visualized with Clarity Western ECL Substrate.

\section{Library Generation}

After the immunization procedure, blood samples were collected $(100 \mathrm{ml})$, peripheral blood lymphocytes (PBLs) were enriched by gradient centrifugation from blood and total RNA was isolated from the PBLs to prepare cDNA (RNAeasy, Qiagen, San Diego, CA, United States). The open reading frames encoding all immunoglobulin heavy-chains were amplified by RT-PCR with primers. Nb open reading frames were amplified through a nested PCR using primers to generate flanking sequences amenable to homologous recombination into pSEX81 (PR3005, Progen Biotechnik GmbH, Heidelberg, Baden- Wuerttemberg, Germany). PCR products $(10 \mu \mathrm{g})$ were mixed with NcoI/ BamHI linearized pSEX81 vector (Progen, $10 \mu \mathrm{g}$ ) and ligated by with T4 DNA ligase and electro-transformed into competent E. coli TG1 cells. Transformants were grown in $2 \mathrm{TY}$ medium containing $2 \%$ glucose and $100 \mu \mathrm{g} / \mathrm{ml}$ ampicillin at $37^{\circ} \mathrm{C}$ overnight (Sabir et al., 2014).

\section{Library Selections}

The transformed TG1 cells were incubated with hyperphage (PRHYPE, Progen Biotechnik GmbH, Heidelberg, BadenWuerttemberg, Germany). The phage particles presenting the VHH library on their tips were collected. Phages containing the $\mathrm{Nb}$ fragments were enriched with solid phase panning. As a negative selection, Enzyme-Linked Immunosorbent Assay (ELISA) wells were coated with $5 \mu \mathrm{g}$ of $\mathrm{KLH}$ at $4^{\circ} \mathrm{C}$ and phage particles were added to the wells and incubated at room temperature for $1.5 \mathrm{~h}$. The unbound phage was moved to another round of negative selection for a total of three rounds. Afterwards, unbound phage were moved to positive selection with nitrated KLH. Bound phages were eluted with $0.1 \mathrm{M}$ triethylamine and used for reinfection of TG1 cells, which were then used for one round of negative selection with WT $14-3-3 ß$ bound by the $(6 \mathrm{x})$ His to Pierce ${ }^{\circledR}$ Nickel Coated Plates (ThermoScientific). The unbound phage were used for two subsequent panning rounds of positive sections with nitroTyr 14-3-3ß immobilized to the $\mathrm{Ni}$ coated plates resulting two full length $\mathrm{Nb}$ sequences (Nb-G5 and $\mathrm{Nb}-\mathrm{F} 110)$.

\section{Nb Validation With Dot Blots}

Immobilon-FL PVDF Membrane was activated by a brief incubation in methanol then equilibrated in MilliQ water followed by equilibration in Towbin's buffer [ $25 \mathrm{mM}$ Tris, $192 \mathrm{mM}$ glycine, 20\% (v/v) methanol ( $\mathrm{pH}$ 8.3)]. Three microlitres of 14-3-3 targets were blotted onto the PVDF membrane obtaining final masses ranging from 0.5 to $5 \mu \mathrm{g}$. The membrane was incubated briefly in Ponceau $S$ to visualize the protein load. The membrane was then rinsed with TBST and blocked with $5 \%$ milk for 1 hour then rinsed $3 \times 5$ min with TBST. Nbs were prepared in TBS to achieve a 1:3,000 desired dilution. $\mathrm{Nb}$ was incubated with the PVDF membrane at RT at times ranging from $1 \mathrm{~h}$ to overnight. After incubation, membranes were rinsed $3 \times 5$ min with TBST. The secondary antibody, horseradish peroxidase (HRP) conjugated antillama, was diluted 1:20,000 in 5\% milk and incubated with the PVDF membrane overnight. Bound Nbs were incubated for $1 \mathrm{~h}$ at room temperature with an anti-llama HRP conjugated secondary antibody diluted $1: 20,000$ in 5\% nonfat milk/TBST. The membranes were washed three times with TBST and visualized with Clarity Western ECL Substrate.

\section{$\mathrm{Nb}$ Validation With Enzyme-Linked Immunosorbent Assay}

Nbs were prepared in TBS to achieve a 1:3,000 desired dilution and were incubated with immobilized WT 14-3-3 (5 $\mu \mathrm{g})$ and $\mathrm{nY}$ $130 / 133$ and nY 213/216 14-3-3 (0.1-5 $\mu \mathrm{g})$ in Ni-coated ELISA wells. Nbs were incubated at room temperature for 1 hour and was visualized after washing $10 \mathrm{x}$ with TBST. Bound Nbs were incubated for $1 \mathrm{~h}$ at room temperature with an anti-llama HRP conjugated secondary antibody diluted 1:1,000 in 5\% nonfat milk/TBST. The wells were washed ten times with TBST and visualized with Clarity Western ECL Substrate.

\section{Nb Validation With Biolayer Interferometry}

All BLI measurements were made on a fortéBIO (Menlo Park, CA, United States) Octet Red96 system using streptavidin (SA) or nickel (Ni) sensors. Assays were performed in 96-well microplates at $37^{\circ} \mathrm{C}$. All sample volumes were $200 \mu \mathrm{L}$. SA sensors were loaded with 14-3-3ß homogeneously biotinylated at the $\mathrm{N}$-terminus $(0.020 \mu \mathrm{g} / \mu \mathrm{L})$. Preliminary assays were done to determine an appropriate amount of biotinylated 14-3-3 to be loaded on the SA sensors, defined as the lowest amount of 14-3-3 that would provide acceptable signal above background for the lowest concentration of $\mathrm{Nb}-\mathrm{G} 5$ as well as reasonable signal with minimal distortion at near-saturating concentrations of Nb-G5. After loading biotinylated 14-3-3ß onto SA sensors, a baseline

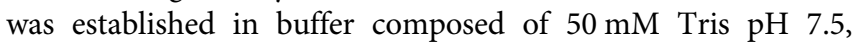




\section{A}

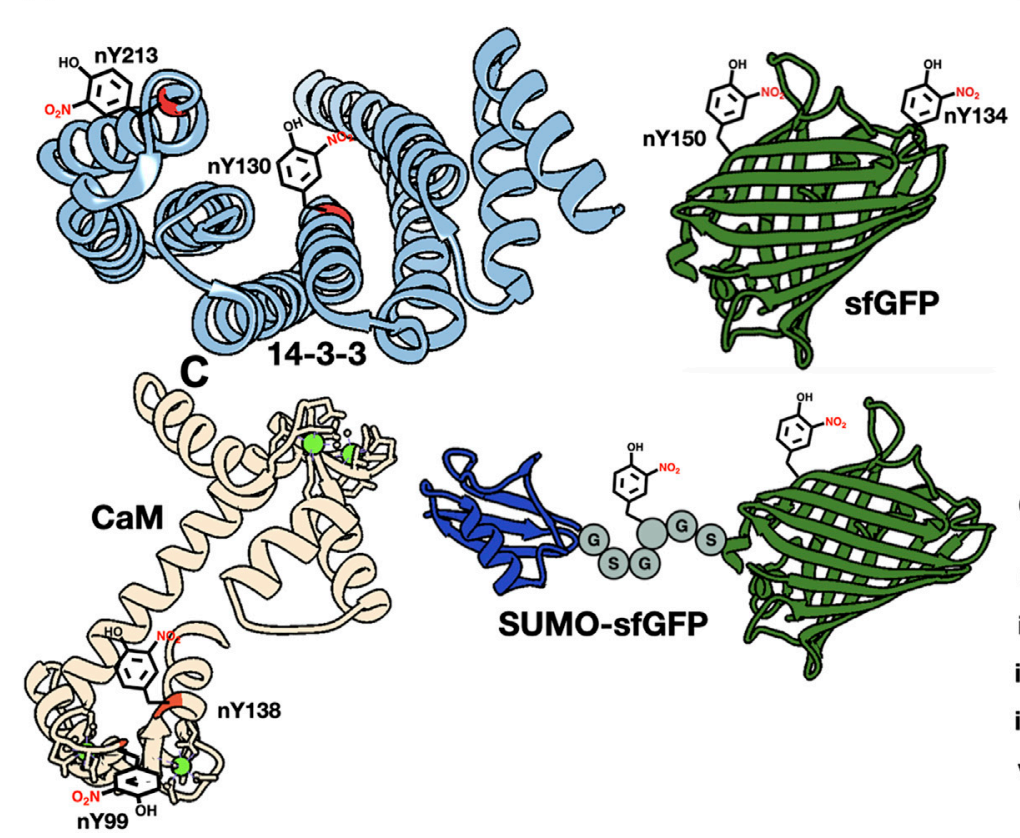

B

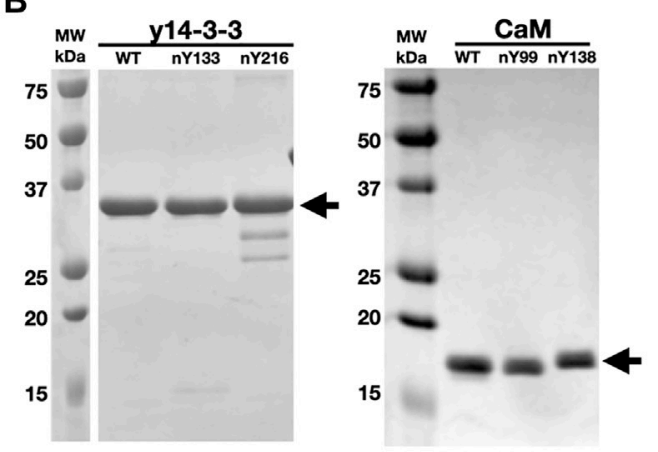

\section{Immunostimulent}

i CaMnY99 -CKDGNG(nY)ISAAE

ii $\mathrm{CaM}_{\mathrm{nY} 138}-\mathrm{CIDGDGQVN}^{\mathrm{n} Y)} \mathrm{EE}$

iii 14-3-3nY133-CKMKGDYHR(nY)LAEFSSGDARE

iv Gen-nY -CNP(nY)PQQP(nY)PQQP(nY)PQQP(nY)EN

v Nitrated KLH

FIGURE 1 | The generation of nitrated molecules for Nb selections and evaluations. (A) Structures of nitrated targets used in this study and generated with Chimera. Nitration sites are indicated with nitroTyr drawing. (PDB ID-2BQ0, 3CLN, 2B3P). (B) SDS-PAGE analysis of purified nitrated targets. (C) Schematic of five immunogens included in injection mixture. Peptides i-iv are conjugated to $\mathrm{KLH}$ with a N-terminal cysteine residue (blue). NitroTyrs in peptides are indicated in red.

$150 \mathrm{mM} \mathrm{NaCl}$ and BSA $(0.1-1 \mathrm{mg} / \mathrm{ml})$ or Tween $(0.05 \%)$. Association with the $\mathrm{Nb}$ was then carried out in the same buffer for $60-90 \mathrm{~s}$ at $\mathrm{Nb}$ concentrations that ranged from $0.0125-1.5 \mu \mathrm{M}$. Dissociation was subsequently measured the same buffer for 120-300 s. BSA was used in all buffers to reduce non-specific binding. These experiments were repeated a minimum of three times, with at least three different preparations of $\mathrm{Nb}-\mathrm{G} 5$.

\section{Statistical Analysis of Biolayer Interferometry Fits}

Data were reference-subtracted and aligned with each other in the Octet Data Analysis software (FortéBIO). Sensograms were fit with a 1:1 binding model to obtain kinetic binding constants. Equilibrium dissociation constant $\left(K_{\mathrm{D}}\right)$ values were calculated from the ratio of $K_{\text {off }}$ to $K_{\text {on }}$. Global fits with an $R^{2}$ higher than 0.98 were considered acceptable.

\section{Crosslinking Reactions}

Crosslinking reactions were conducted in the following manner. azidoPhe-incorporated protein and target were

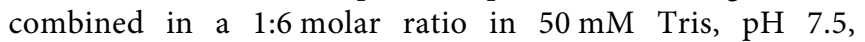
$150 \mathrm{mM} \mathrm{NaCl}$ for a total reaction volume of $50 \mu \mathrm{L}$. This solution was allowed to incubate for $30 \mathrm{~min}$ on ice, and then exposed to UV light $(254 \mathrm{nM})$ via a UVP Inc. Model UVGL-25 Multiband UV-254/366 nm Mineralight Lamp for 5 minutes in a 96 well UV-transparent plate also on ice. After exposure to UV light, the solutions were removed from the wells and mixed with SDS sample loading buffer and analyzed with SDS-PAGE.

\section{Western Blots}

Western blot samples were separated on 4-22\% gradient SDSPAGE gels, transferred to PVDF membrane ( $30 \mathrm{~V}$, overnight) blocked with Licor blocking buffer in TBST, and probed with anti-His (Takara, 1:1,000), or anti-V5 (Invitrogen, 1:500) primary antibodies rocking for $16 \mathrm{~h}$ at $4^{\circ} \mathrm{C}$. After rinsing three times with TBST, the membranes were than incubated with Li-Cor IRDye $800 \mathrm{CW}$ Goat anti-rabbit or anti-mouse IgG $(1: 10,000)$ secondary antibody, rocking for $1 \mathrm{~h}$ at room temperature, and washed three times for $5 \mathrm{~min}$ in TBST. The membrane was then scanned using a Li-Cor Odyssey 9,120 Imaging System. If antibody overlays were desired, after imaging, the additional antibody was added to the membrane and incubated for $1 \mathrm{~h}$ at room temperature. After incubation, an appropriate secondary antibody was added, and the blot visualized as described above.

\section{RESULTS}

\section{Strategy and nitroTyr Protein Generation Strategy}

With the goal of generating a $\mathrm{Nb}$ that is either selective for a nitration site in a specific protein or one that binds nitroTyr regardless of protein context, we chose to immunize an animal with peptides that represented multiple nitrated targets (general and protein-specific) and use the results of a preliminary serum screen to decide the best target(s) to pursue. Per usual protocols 
TABLE 1 | NitroTyr proteins produced for Nb evaluation.

\begin{tabular}{|c|c|c|c|c|}
\hline Protein & Nitration sites & Biological significance & Structural context & Ref \\
\hline$y 14-3-3$ & Y133 & Nitrated in vivo & $\begin{array}{l}\text { Key component of pSer/pThr recognition } \\
\text { triad }\end{array}$ & $\begin{array}{l}\text { Zhao et al. (2017), Pennington et al. } \\
\text { (2018) }\end{array}$ \\
\hline$y 14-3-3$ & Y216 & Nitrated in vivo & Solvent exposed & $\begin{array}{l}\text { Nuriel et al. (2015), Pennington et al. } \\
\text { (2018) }\end{array}$ \\
\hline h14-3-3 & Y130 & Nitrated in vivo & $\begin{array}{l}\text { Key component of pSer/pThr recognition } \\
\text { triad }\end{array}$ & $\begin{array}{l}\text { Zhao et al. (2017), Pennington et al. } \\
\text { (2018) }\end{array}$ \\
\hline h14-3-3 & Y213 & Nitrated in vivo & Solvent exposed & $\begin{array}{l}\text { Nuriel et al. (2015), Pennington et al. } \\
\text { (2018) }\end{array}$ \\
\hline CaM & Y99 & Nitration site is a target for denitrase & Found in globular $\mathrm{Ca}^{2+}$ binding domain & $\begin{array}{l}\text { Corti et al. (1999), Smallwood et al. } \\
\text { (2007) }\end{array}$ \\
\hline CaM & Y138 & $\begin{array}{l}\text { Nitration at site modulates signaling } \\
\text { behavior }\end{array}$ & Found in a conformationally dynamic region & $\begin{array}{l}\text { Mukherjea et al. (1996), Porter et al. } \\
\text { (2020) }\end{array}$ \\
\hline GFP & Y134 & None & In protein loop & $\mathrm{N} / \mathrm{A}$ \\
\hline GFP & N150 & None & On side of GFP barrel & $\mathrm{N} / \mathrm{A}$ \\
\hline $\begin{array}{l}\text { SUMO- } \\
\text { GFP }\end{array}$ & $\begin{array}{l}\text { Link, N150 (2x } \\
\text { nitroTyr) }\end{array}$ & None & $\begin{array}{l}\text { Dual nitration sites; one on a disordered } \\
\text { linker }\end{array}$ & $\mathrm{N} / \mathrm{A}$ \\
\hline
\end{tabular}

(Vincke et al., 2012), from the blood of a seropositive immunized animal, we generated a phage-display library from peripheral blood lymphocyte RNA and carried out phage display selections with select nitrated target proteins. The selected nitroTyr-specific Nbs were then characterized for their affinity and selectivity using both semi-quantitative (ELISA, dot-blot) and quantitative (biolayer interferometry) methods.

\section{NitroTyr-Protein Targets for Selections and for Immunizations}

We selected signaling hub proteins 14-3-3 and CaM as nitroTyr-protein targets (Figure 1A). The yeast ortholog of 14-3-3 (commonly known as BMH1 and referred to here as "y14-3-3" for clarity) was used initially as it is a functional model for human 14-3-3 [(Clapp et al., 2012), 55\% identity, h14-3-3] and we had established expression and purification systems for it. While proteomics studies have identified multiple sites of in vivo nitration of y14-3-3 (Nuriel et al., 2015; Pennington et al., 2018), we initially focused on Y133, which is a key part of pSer/pThr binding site and fully conserved in all eukaryotic 14-3-3 proteins. For CaM, we focused on Y99 which when nitrated serves as a biomarker of oxidative stress (Smallwood et al., 2003) and Y138 which both enhances CaM binding to eNOS and has been shown to be transient in activated macrophages (Porter et al., 2020). These two sites also provide contrasting protein contexts, as Y99 is found in the less solvent-exposed globular $\mathrm{Ca}^{2+}$-binding domain and Y138 is contained in a conformationally dynamic region (Smallwood et al., 2007; Piazza et al., 2012; Porter et al., 2020) (Figure 1A). For our immunization series, we designed peptides encompassing all three of these nitration sites along with an additional peptide containing several nitroTyrs to enhance the chance of obtaining Nbs that were generally specific for nitroTyr. With these peptides, we also included in the immunization series chemically nitrated keyhole limpet hemocyanin (KLH) (Figure 1C) which emulated the successful strategy employed for the original nitroTyr-Ab generation (Beckmann et al., 1994).
NitroTyr-Proteins for Further Binding Characterization In addition to nitrated immunogens and the specific target proteins to be used in selections, we generated some nitroTyrproteins to further characterize the specificities of the generated Nbs (Figure $\mathbf{1 A}$ and Table 1). To that end, we identified y14-3-3 Y216 whose nitration is also physiologically relevant and is more solvent-exposed than Y133. Additionally, the human variants of 14-3-3 $\beta$ (nY130 and nY213) were used because their nitration is more relevant to human disease. We further made superfolder green fluorescent proteins (sfGFPs) with nitroTyr inserted in the place of N150 or D134 to study the ability of the $\mathrm{Nb}$ to bind a nitroTyr that juts out from a smooth protein surface (site 150) as compared to a nitroTyr located in the loops of the barrel (site 134). And finally, to evaluate the effects of having multiple sites of nitration contained in a single protein, we created a SUMO-linkersfGFP containing two sites of nitration. Using GCE, we produced all full-length, homogenously nitrated proteins, purified to $>95 \%$ purity with yields ranging from $30-45 \mathrm{mg} /$ L of culture (Figure 1B). The fidelity of site-specific nitroTyr incorporation was verified by mass spectrometry, with correct masses obtained for both unmodified and nitrated targets (Supplementary Figure S1). In total, nine specific nitroTyr proteins were produced for generating and testing Nbs (Table 1).

\section{Identification of Two Promising Nbs Selected to Recognize 14-3-3 nY133} Immune-Serum Generation and Screening

All five nitrated immunogens were simultaneously injected into an alpaca following a standard immunization procedure (Materials and Methods). To monitor the production of Camelid antibodies that target nitroTyr-proteins, pre- and post-injection sera (collected before and after full immunization) were incubated with membrane-immobilized y14-3-3 and CaM targets. Development of the membranes revealed bands in the lane containing $y 14-3-3_{\mathrm{nY} 133}$ and a faint 

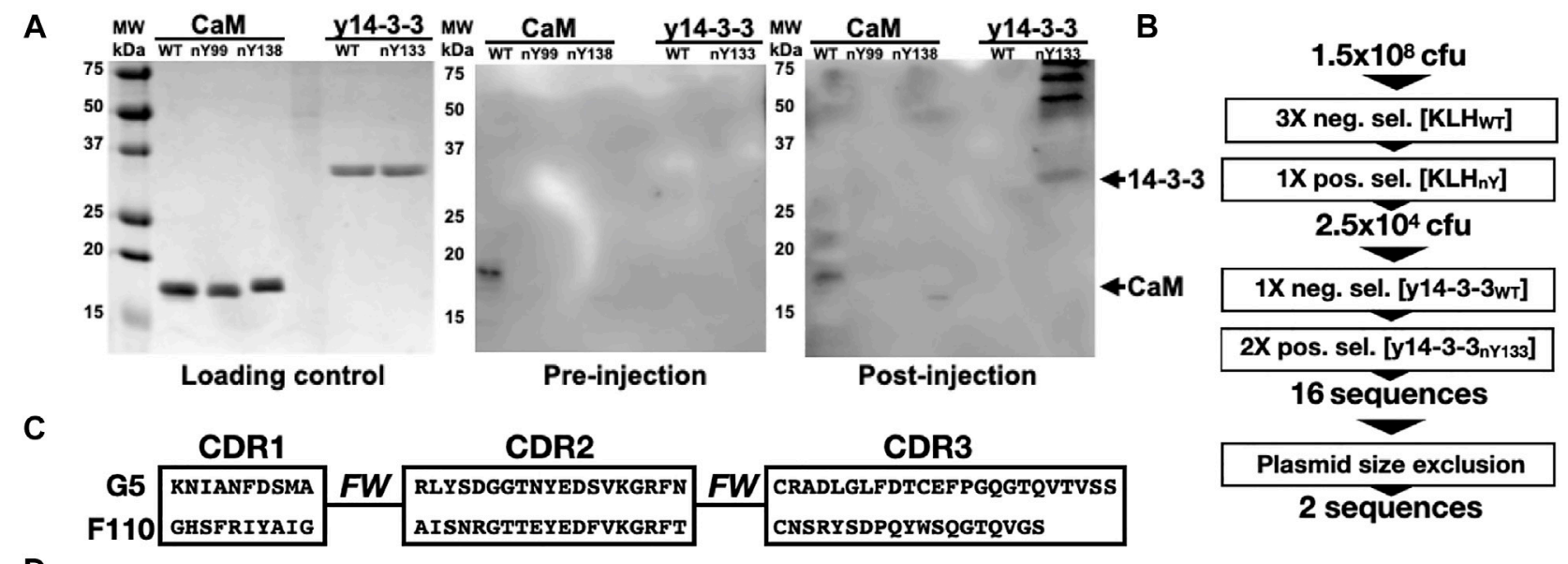

D
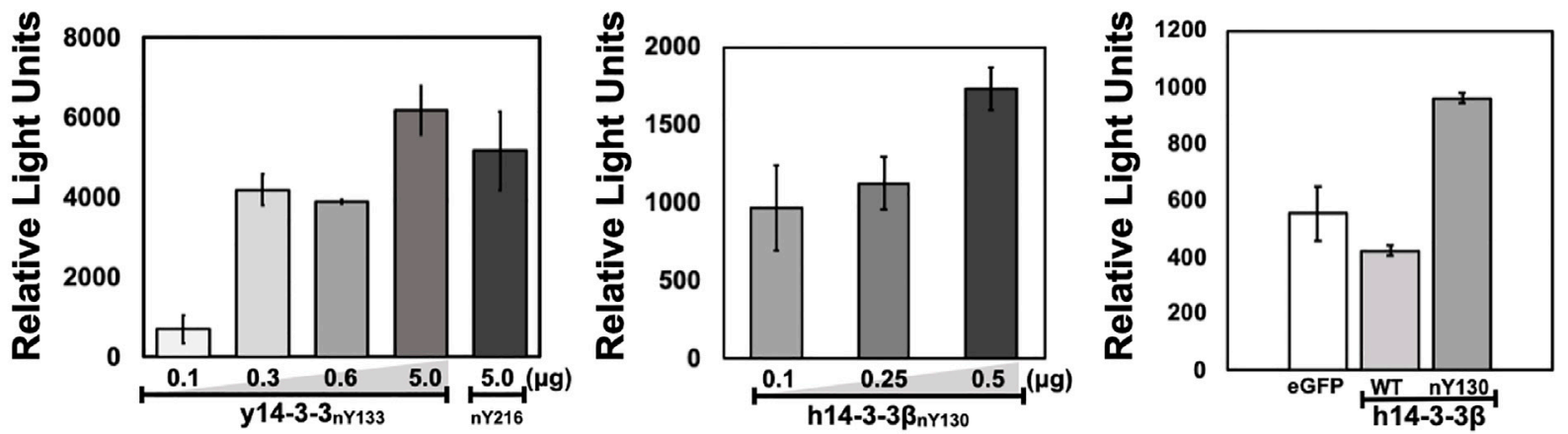

FIGURE 2 | Selection and preliminary validation of Nb-G5. (A) Serum analysis of injected animal against nitrated targets. Five protein targets (WT and nitroTyr, listed at top of gel) were resolved with SDS-PAGE. Gels were either stained (Loading control) or transferred to a PVDF membrane and incubated with serum from before injections (pre-injection) or after (post-injection). These membranes were probed with HRP-conjugated anti-llama primary and visualized. Arrows indicate the expected migration of protein targets. (B) Schematic of selections with full-length, folded nitroTyr 14-3-3. Included is the number of cfu or sequences obtained after significant rounds. (C) CDR regions of Nb-G5 and Nb-F110. "FW" indicates the framework region of the Nbs. Full sequences are found in Supplemental Figure S2. (D) ELISA of Nb-G5 nitrated 14-3-3. Increasing amounts of His-tagged y14-3-3 $\beta$ nitroY133 (left panel) 14-3-3 $\beta$ nitroY130 (middle panel) were immobilized on a nickel-coated ELISA plate and incubated with NB-G5 (25 $\mathrm{mg} / \mathrm{ml})$ in TBST followed by incubation with HRP-conjugated anti-llama primary antibody. Right panel: His-tagged sfGFP, $14-3-3 \beta$

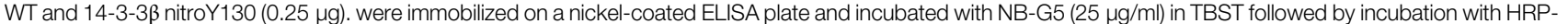
conjugated anti-llama primary antibody.

band in a lane containing $\mathrm{CaM}_{\mathrm{nY} 138}$, none of which appear on the pre-serum membrane providing evidence that antibodies specific to nitrated targets had been produced (Figure 2A). Additional prominent higher molecular weight bands were also visible in the y14-3-3 ${ }_{\text {nY133 }}$ lane; these may represent camelid antibody affinity for dimerized forms of 14-3-3. Due to the intensity of the y14-3$3_{\mathrm{nY} 133}$ bands compared to the other targets $\left(\mathrm{CaM}_{\mathrm{nY} 99}\right.$ and $\mathrm{CaM}_{\mathrm{nY} 138}$ ), we sought to develop a protein and nitration sitespecific $\mathrm{Nb}$ and pursued selections against y14-3-3 $3_{\mathrm{nY} 133}$.

\section{Library Generation and Phage Display Selections Using y14-3-3 $3_{\mathrm{nY} 133}$}

To generate a phage display library, the total RNA from peripheral blood lymphocytes (PBLs) after the immunization procedure were used to make $\mathrm{Nb}$-encoding cDNA. The cDNAs were amplified and subcloned into the phagemid vector pSEX81, generating a phage display library. With this library, we performed seven rounds of phage display selections
(Figure 2B). The first three rounds were negative selections using $\mathrm{KLH}$ as the target, designed to exclude anti-KLH library members. Then, mimicking the process that yielded the initial nitroTyr-Abs (Beckmann et al., 1994), we carried out a positive selection with nitroTyr-KLH to ensure that remaining Nbs had some direct recognition of nitroTyr. These four rounds of selection diminished the library size from $1.5 \times 10^{8} \mathrm{cfu}$ (colony forming units) to $2.5 \times 10^{4} \mathrm{cfu}$ (Figure 2B).

We then panned for library members that were selective for y14-3-3 $3_{\text {nY133 }}$ by using one round of negative selection with wild type y14-3-3, followed by two rounds of positive

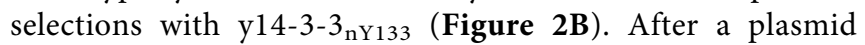
size-exclusion step to eliminate truncated $\mathrm{Nbs}$, two fulllength Nbs were obtained: Nb-G5 and Nb-F110 (Figure 2C, Supplementary Figure S2). Expression and purification of $\mathrm{Nb}-\mathrm{G} 5$ yielded protein sufficient for downstream characterization (Supplementary Figure S3) but we were unable to identify suitable $E$. coli expression strategies for 
Nb-F110. As a result, we moved forward with evaluating only $\mathrm{Nb}-\mathrm{G} 5$.

\section{Characterization of the Binding Properties of Nb-G5}

Semi-Quantitative Survey of Nb-G5 Binding to 14-3-3 Protein Forms

Binding of Nb-G5 was qualitatively evaluated with both dot-blot assays and enzyme-linked immunosorbent assays (ELISA). With the dot-blot assay, we evaluated Nb-G5 selectivity and $\mathrm{Nb}$ binding sensitivity. Nitrated targets y14-3-3 ${ }_{\mathrm{nY} 133}$ and y14-3$3_{\text {nY216 }}$ along with y14-3-3 $\beta_{W T}$ were dotted in varying amounts onto a membrane and probed with Nb-G5; binding was observed

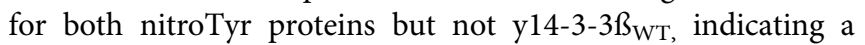
selectivity for nitrated protein (Supplementary Figure S4). y14-3-3 ${ }_{\text {nY216 }}$ was included to see if Nb-G5 possessed any sensitivity for nitration independent of the protein context of site nY133. Having at this point successfully expressed the more biomedically relevant nitrated human 14-3-3ß ( $h 14-3-3 \beta)$ isoforms, we also probed these and found they were recognized with a similar level of sensitivity (Supplementary Figure S4).

The range of selectivity and sensitivity of Nb-G5 to immobilized, folded protein in solution was assayed by ELISA. For both the yeast and human 14-3-3 variants, significant signal above background was observed, down to $0.1 \mu \mathrm{g}$ of immobilized nitrated 14-3-3. The selectivity of Nb-G5 for nitroTyr was evaluated by assaying immobilized $14-3-3 \beta_{\mathrm{WT}}$ and sfGFP (as a negative control) alongside h14-3-3 $\beta_{\mathrm{nY} 130}$. Significant signal for h14-3-3ß $\beta_{\text {nY130 }}$ over sfGFP and h14-3-3ß wT, which both exhibited a similar level of background was also observed by ELISA (Figure 2D). Based on this, we decided to carry out quantitative binding studies with the h14-3-3ß forms rather than with the y14-3-3 forms.

\section{Quantitative Analyses Nb-G5 Affinity and Selectivity}

Encouraged by our preliminary evaluation of $\mathrm{Nb}$ binding, we employed biolayer interferometry (BLI) to quantitatively assess the sensitivity and selectivity of Nb-G5. Avi-tagged h14-3-3ß variants (WT, nY130 and nY213) were immobilized onto BLI tips and incubated with $\mathrm{Nb}-\mathrm{G} 5$ to measure the association and dissociation kinetics of binding. Global fits of the BLI sensograms in $0.25 \%$ bovine serum albumin (BSA) gave a $\mathrm{K}_{\mathrm{D}}$ value for Nb-G5 against h14-3-3 $\beta_{\mathrm{nY} 130}$ of $\sim 14 \mathrm{nM}$, a value that is an order of magnitude tighter than that obtained for Nb-G5 against h14-3-3 $\beta_{\mathrm{WT}}$ under the same assay conditions, and 2-5 times tighter than the $K_{D}$ for h14-3-3 $\beta_{\mathrm{nY} 213}$ (Figure 3A and Table 2). The main contributor to the observed differences in $\mathrm{K}_{\mathrm{D}}$ is the dissociation rate, consistent with the nitroTyr protein forms making specific interactions with Nb-G5 that slow its dissociation (Table 2). To minimize the contribution of non-specific binding, the assays were also performed in $1 \%$ BSA. In these experiments, binding to h14-3-3 $\beta_{\mathrm{WT}}$ was almost completely ablated and kinetic constants could not be determined, although binding to h14-3-3 $\beta_{\mathrm{nY} 130}$ was still observed $\left(K_{D} \sim 40 \mathrm{nM}\right.$, Figure 3B$)$. This is consistent with binding to WT protein being non-specific, whereas binding to both nitrated protein forms involving some level of specific recognition.

To further assess the relative contributions of the nitroTyr group itself versus the protein context to the binding of Nb-G5 for nitrated targets, we tested Nb-G5 binding with proteins containing nitroTyr in different protein locations (Figure 1A). Proteins included three CaM forms (WT, nY99, nY138 in 0.05\% Tween) and four sfGFP forms [WT, nY134, nY150, SUMO-linksfGFP (2x nitroTyr) in $0.2 \%$ BSA, Table 1]. Although none of these proteins bound as well as either of the nitrated forms of h143-3ß, local fits revealed some binding to both $\mathrm{CaM}_{\mathrm{nY} 138}$ (Figure 3C panel A), sfGFP ${ }_{n Y 134}$ and SUMO-link-sfGFP (2x nitroTyr) (Figure 3C panel B) for these targets at high Nb-G5 concentrations $(>200 \mathrm{nM})$. These interactions generated $K_{D}$ values $>100 \mathrm{nM}$, and no notable binding to the wild type proteins or to the nitrated targets $\mathrm{GFP}_{\mathrm{nY} 150}$ or $\mathrm{CaM}_{\mathrm{nY} 99}$ was observed (Supplementary Figure S5).

\section{Nb-G5 Nanobody as Basis for Creating nitroTyr Specific Cross-Linking Tools Identification of Potential Sites and Construct Design for Crosslinking Experiments}

As noted in the introduction, generating covalent $\mathrm{Nb}$ binders (i.e., "GlueBodies") through incorporating ncAAs with crosslinking abilities (ncAA-CL), is a means to target proteins for degradation (Zhang et al., 2021). Nbs that target oxPTMs for degradation would be powerful tools for discovering possible physiological changes triggered by specific oxidized proteins. A variety of ncAA-CL can be incorporated by GCE, including chemically reactive, proximity-induced and photo-reactive ncAA-CLs (Figure 4A). Because the GCE machinery for photocrosslinking ncAA-CL 4-azido phenylalanine (azidoPhe, $\mathrm{pAzF}$ ) is robust, the ncAA is commercially available and the crosslinking assays are simple to perform, we decided to investigate the suitability of $\mathrm{Nb}-\mathrm{G} 5$ as a covalent crosslinker by the installation of azidoPhe into Nb-G5 with GCE.

We first sought to identify locations on Nb-G5 near the edge of the paratope, so that upon binding they would be close to the target, yet not interfere with $\mathrm{Nb}$ binding itself. We noticed the CDR3 loop of Nb-G5 contained an unpaired cysteine residue which is unusual in Nbs (Bannas et al., 2017) and so we speculated this Cys was part of the antigen binding site. To evaluate its contribution, we generated a C115S mutant of Nb-G5 and observed by BLI that the binding to h14-3-3$\beta_{\text {nY130 }}$ decreased by six-fold, and that the selectivity for h14-3-3 $\beta_{\text {nY130 }}$ over h14-3$3_{\text {WT }}$ was lost (Figure 4B).

We created two different strategies to visualize the crosslinked material (Figure 4C). The first was appending a C-terminal GFP fusion to the Nb-G5, which would give a sensitive fluorescent signature to the $\mathrm{Nb}$ and allow CL-dependent mass shifts to be easily observed with in-gel fluorescence. Concerned that a bulky GFP tag could impact the binding of Nb-G5, we also designed a construct with a smaller C-terminal V5 tag, which could be visualized by Western blotting (Figure 4C, "ii"). Also, since we expected the Nb-G5 incorporating azidoPhe would express 


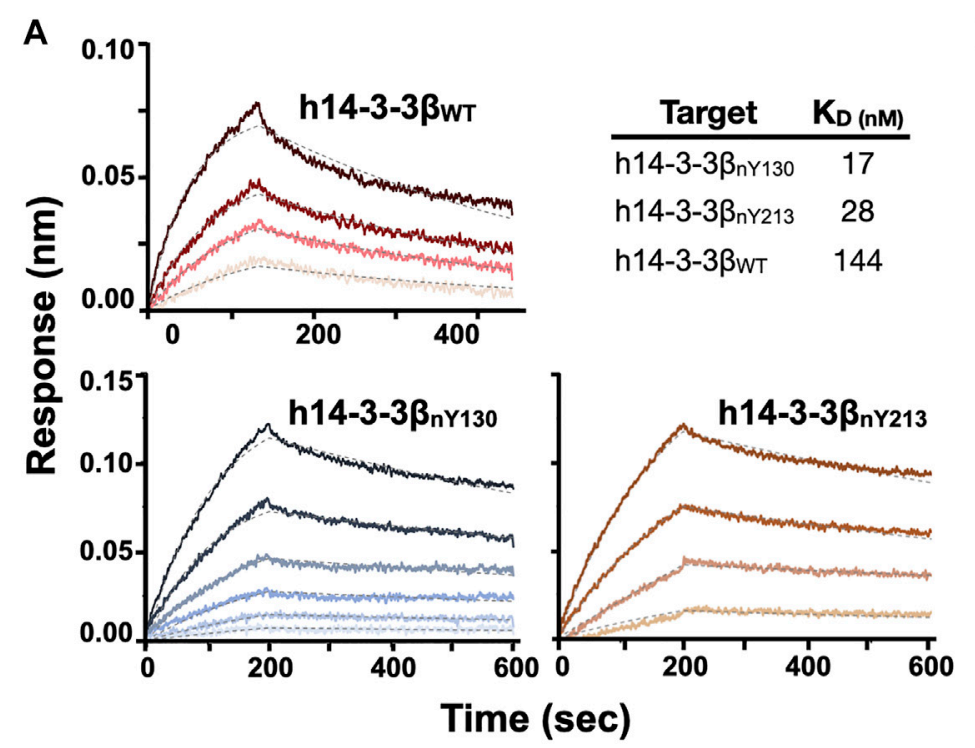

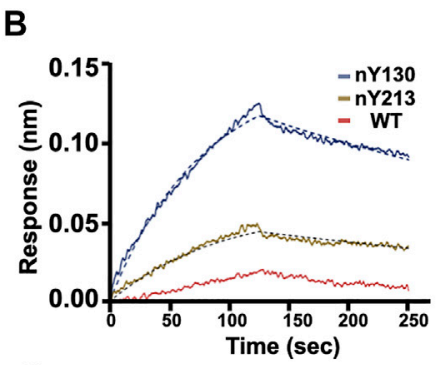

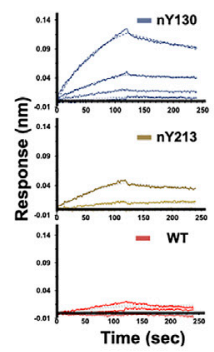

C

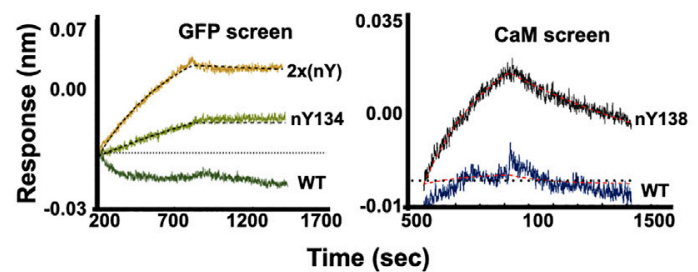

FIGURE 3|BLI analysis of Nb-G5 binding ability. (A) BLI sensograms of Nb-G5 against nitrated and WT 14-3-3 3 in 0.2\% BSA. For global fits, concentrations of NbG5 against nitrated ranged from 25 to $200 \mathrm{nM}$ for $14-3-3 \beta_{\mathrm{nY} 130}, 40-200 \mathrm{nM}$ for $14-3-3 \beta_{\mathrm{nY} 213}$ and $270-900 \mathrm{nM}$ for $14-3-3 \beta_{\mathrm{WT}}$. $\mathrm{K}_{\mathrm{D}}$ values for global fits are listed in the upper right. (B) BLI sensograms of Nb-G5 against nitrated and WT 14-3-3 $\beta$ in 1\% BSA. Left panel: the sensograms for all three targets at 450 nM. Right panel: Global fits for Nb-G5 in 1\% BSA ranging from 80 to 450 nM. (C) Left panel: Local fits of Nb-G5 (200 nM) against sfGFP WT, sfGFP $_{n Y 134}$, and sfGFP $2 \times n Y$. Right panel: Local fits of $\mathrm{Nb}-\mathrm{G} 5(300 \mathrm{nM})$ against $\mathrm{CaM}_{\mathrm{WT}}$ and $\mathrm{CaM}_{\mathrm{nY} 138}$.

TABLE 2 | Kinetic constants for Nb-G5:14-3-3 binding.

\begin{tabular}{|c|c|c|c|c|}
\hline TARGET (in $0.2 \%$ BSA) & $K_{D}(n M)$ & $k_{\text {on }}\left(10^{4} M^{-1} \mathrm{~s}^{-1}\right)$ & $k_{\text {off }}\left(10^{3} \mathrm{~s}^{-1}\right)$ & BSA \\
\hline $14-3-3 \boldsymbol{\beta}_{\mathrm{nY} 130}$ & $17 \pm 3$ & $2.2 \pm 0.2$ & $0.33 \pm 0.02$ & $0.2 \%$ \\
\hline $14-3-3 \boldsymbol{\beta}_{\mathrm{nY} 213}$ & $28 \pm 2$ & $2.6 \pm 0.1$ & $0.73 \pm 0.02$ & $0.2 \%$ \\
\hline $14-3-3 \beta_{W T}$ & $144 \pm 8$ & $2.1 \pm 0.6$ & $2.3 \pm 0.5$ & $0.2 \%$ \\
\hline TARGET (1.0\% BSA) & $K_{D}(n M)$ & $k_{\text {on }}\left(10^{4} M^{-1} \mathrm{~s}^{-1}\right)$ & $k_{\text {off }}\left(10^{3} s^{-1}\right)$ & BSA \\
\hline $14-3-3 \boldsymbol{\beta}_{\mathrm{nY} 130}$ & $36 \pm 3$ & $3.2 \pm 0.1$ & $1.19 \pm 0.07$ & $1.0 \%$ \\
\hline $14-3-3 \boldsymbol{\beta}_{\mathrm{nY} 213}$ & $110 \pm 3$ & $2.05 \pm 0.02$ & $2.26 \pm 0.05$ & $1.0 \%$ \\
\hline $14-3-3 \boldsymbol{\beta}_{\mathrm{WT}}$ & Undeterminable & Undeterminable & Undeterminable & $1.0 \%$ \\
\hline
\end{tabular}

less well, we sought to enhance the expression through adding a cleavable N-terminal bdNEDD8 solubility tag (Pleiner et al., 2018). With the systems designed, we selected six potential sites flanking $\mathrm{C} 115$ for azidoPhe incorporation, and all six sites for both designs (GFP and V5) yielded sufficient cleaved, azidoPhe-containing $\mathrm{Nb}-\mathrm{G} 5$ for crosslinking experiments (Figure 4D, Supplementary Figure S6).

\section{Crosslinking Attempts With h1413-3 $B_{\mathrm{nY} 133}$ and $\mathrm{CaM}_{\mathrm{nY} 138}$}

As nitroTyr-protein targets, we selected the two that were bound best by Nb-G5: 14-3-3 $3_{\mathrm{nY} 133}$ and $\mathrm{CaM}_{\mathrm{nY} 138}$. Both targets possess C-terminal His tags, allowing them to be detected with Western Blot. Screening of the six azidoPhe sites with both nitrated targets for their ability to yield nitroTyr-dependent crosslinked products yielded disappointing results (Supplementary Figures S7, S8). Some bands appear at the correct molecular weight range for pAzF113 with the CaM target and pAzF108 and pAzF110 for the
14-3-3 target, but even for these the amount of CL product is minimal and masked by the presence of non-specific crosslinked products. In addition, the photoactivation appears to dimerize the nitroTyr-containing target protein, further complicating analysis and making the approach with this Nb-G5 and azidoPhe photocrosslinker challenging to implement in a more complex environment (live cell).

\section{DISCUSSION}

With the aid of GCE technology, we show it is possible to generate a $\mathrm{Nb}$ selective for an oxidatively-modified protein target, opening the doors to dynamic studies of oxPTM-impacted proteins in living cells. This technology is not limited to oxPTMs, as GCE can provide site-specifically modified protein for many biologically important PTMs, including phosphoserine (Rogerson et al., 2015; Zhu et al., 2019) and acetyl lysine (Neumann-Staubitz et al., 
A

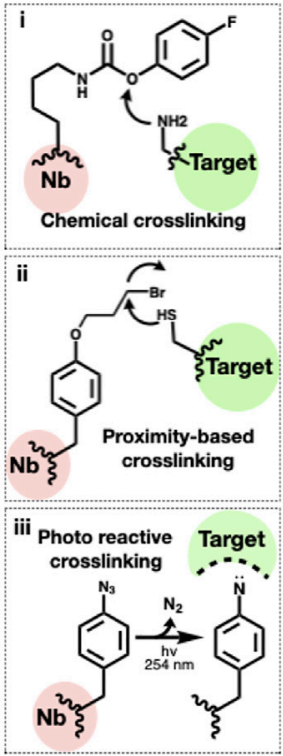

B

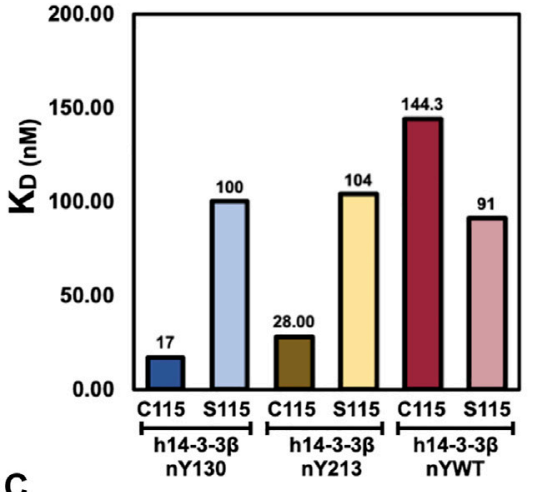

C

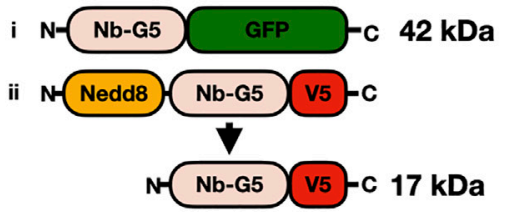

D

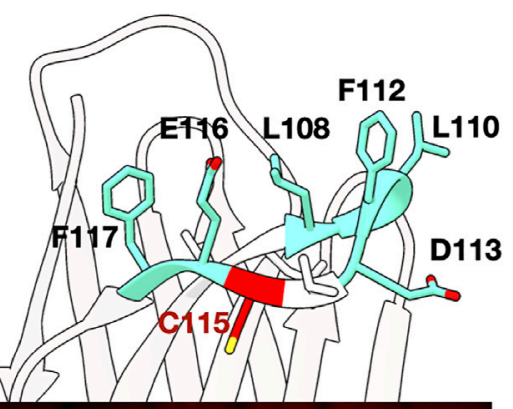

Nb-G5-pAzF-V5

anti-V5

Western

Blot

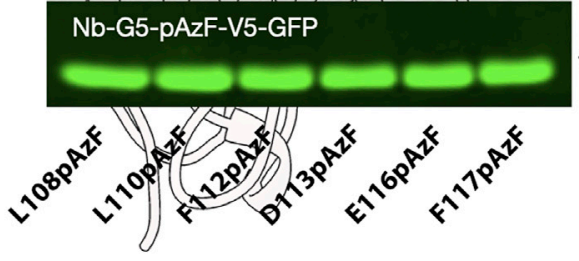

In-gel fluorescent assay

FIGURE 4 | Generating an anti-nitroTyr cross-linking tool. (A). Examples of three common crosslinking strategies: (i) Chemical crosslinkers, such as the carbamatecontaining ncAA FPheK, display a high reactivity to proximal nucleophilic residues (e.g., Lys, Cys, and Tyr) under weakly basic conditions (Xuan et al., 2017); (ii) Proximitybased crosslinkers, such as the haloalkane ncAA shown, react with Cys residues in the target protein facilitated by an increased local effective concentration (Xiang et al., 2014); and (iii) Photoreactive crosslinkers, such as benzophenones, diazirines, and azides (as in the azidoPhe ncAA shown), contain UV-induced cross-linking moieties that react with diverse proximal groups (Hoffmann 2020). (B). KDS of Nb-G5 and Nb-G5 (C115S) against nitrated and WT 14-3-3 are shown. (C). Constructs of Nb-G5-GFP (i) and Nedd8-Nb-G5-V5 (ii). (D). A model of Nb-G5, generated by ABodyBuilder: automated antibody structure predictor (Leem et al., 2016). The CDR3 loop unpaired cysteine (C115) is indicated in red and the adjacent resides individually substituted with azidoPhe are colored teal and labeled. An anti-V5 western blot image (top panel) and an in-gel fluorescence assay (bottom panel) show both kinds of azidoPhe-encoded Nb-G5 constructs expressed well.

2021). This allows, for the first time, a rigorous characterization of the site and protein specificity of Nbs developed against these targets contributing to both the engineering of nanobodies for specific purposes as well our general knowledge of Nb-target recognition.

Starting from the serum of an animal injected with a mixture of five nitrated immunogens, we developed an anti-nitroTyr nanobody library and performed phage-display selections for the nitrated target $14-3-3_{\mathrm{nY} 130}$, obtaining the sequence for a single soluble nanobody, Nb-G5. BLI analysis of Nb-G5 with 14-3-3 $3_{\text {nY130 }}$ revealed a 10-fold selectivity for $14-3-3_{\text {nY130 }}$ over $14-$ $3-3_{\mathrm{WT}}$ in $0.2 \%$ BSA solutions, exhibiting a modest $\mathrm{K}_{\mathrm{D}}$ of $\sim 14 \mathrm{nM}$. In $1 \%$ BSA solutions, $\mathrm{Nb}-\mathrm{G} 5$ exhibited a higher $\mathrm{K}_{\mathrm{D}}$ but complete selectivity for $14-3-3_{\mathrm{nY} 130}$ over $14-3-3_{\mathrm{WT}}$. Nb-G5 also showed some weak yet selective binding to other non-14-3-3 nitrated targets, which may indicate that the $\mathrm{Nb}$ is interacting with the nitroTyr molecule itself. This weak binding was site-dependent and appeared to be strongest when the nitroTyr molecule was exposed, but still surrounded by ample protein context.

After obtaining some information about a cysteine that is critical to $\mathrm{Nb}$ selectivity, we were able to site-specifically install the ncAA azidoPhe in the CDR3 region of Nb-G5. Using robust GCE machinery for azidoPhe, all azidoPhe sites were successfully installed at reasonable yields allowing us to easily screen the library against two nitrated targets for crosslinking. However, the amount of crosslinked product produced was small, and the presence of nitroTyr in proteins appeared to increase the propensity of the nitrated proteins to crosslink under UV exposure, limiting the suitability of this specific crosslinking strategy for nitroTyr targets. Nitrotyrosine increases the UV absorbance of the target proteins (Crow and Beckman 1995) and appears to be competing with the desired photocrosslinking pathway. With GCE, we are not limited to one type of crosslinking chemistry and could utilize crosslinkers that instead are proximity-based and operate independently of UV irradiation, including ncAA with bromoalkyl moieties (Cigler et al., 2017). These would be well-suited for Nbs targeting chemically labile modifications which may be more sensitive to UV irradiation such as those installed under conditions of oxidative stress. Regardless, we were encouraged by the ability to form ncAA-CL encoded-Nbs, showing that GCE-technology is well suited to be implemented in every step of $\mathrm{Nb}$ development, from selections to additional functionalities.

The mid-nanomolar $K_{D}$ obtained for NB-G5 with no additional rounds of affinity maturation is promising, however, $\mathrm{Nbs}$ that are designed to be employed as intracellular tools require low to sub-nanomolar affinities for their targets (Mahajan et al., 2018). Originally, when we were performing the selections, we included a positive selection step with nitrated KLH to emulate the strategy that produced the first anti-nitroTyr antibodies. It may be that more selective binders for 14-3-3nY were excluded from the set in that $\mathrm{KLH}_{\mathrm{nY}}$ positive selection step, although Nb-G5 has slight general specificity. The pre-KLH positive step library still exists, and it represents a valuable 
resource in itself that could be used to select for Nbs against other nitroTyr proteins, or a tighter 14-3-3nY133 binder. With respect to the selections themselves, $\mathrm{Nb}$-selection technology is rapidly improving. With GCE-produced proteins and multiple rounds off affinity maturation, the development of anti-oxPTM Nbs with sub-nanomolar affinities, appropriate of intracellular interrogation, are within reach. Such $\mathrm{Nbs}$ will be powerful tools that will open up a window into the complex intracellular dynamics of oxidatively modified proteins and their impacts.

\section{DATA AVAILABILITY STATEMENT}

The original contributions presented in the study are included in the article/Supplementary Materials, further inquiries can be directed to the corresponding author.

\section{ETHICS STATEMENT}

The animal study was reviewed and approved by Oregon State University Institutional Animal Care and Use Committee.

\section{AUTHOR CONTRIBUTIONS}

Conceptualization: EV, CR, DM, CC, RC, and RM. Methodology: EV, SG, DM, CC, SB, RC, and RM.

\section{REFERENCES}

Bannas, P., Hambach, J., and Koch-Nolte, F. (2017). Nanobodies and NanobodyBased Human Heavy Chain Antibodies as Antitumor Therapeutics. Front. Immunol. 8, 1603. doi:10.3389/fimmu.2017.01603

Bartesaghi, S., and Radi, R. (2018). Fundamentals on the Biochemistry of Peroxynitrite and Protein Tyrosine Nitration. Redox Biol. 14, 618-625. doi:10.1016/j.redox.2017.09.009

Beckmann, J. S., Ye, Y. Z., Anderson, P. G., Chen, J., Accavitti, M. A., Tarpey, M. M., et al. (1994). Extensive Nitration of Protein Tyrosines in Human Atherosclerosis Detected by Immunohistochemistry. Biol. Chem. HoppeSeyler 375 (2), 81-88. doi:10.1515/bchm3.1994.375.2.81

Bery, N., Keller, L., Soulié, M., Gence, R., Iscache, A.-L., Cherier, J., et al. (2019). A Targeted Protein Degradation Cell-Based Screening for Nanobodies Selective toward the Cellular RHOB GTP-Bound Conformation. Cell Chem. Biol. 26 (11), 1544-1558. doi:10.1016/j.chembiol.2019.08.009

Beyer, J. N., Hosseinzadeh, P., Gottfried-Lee, I., Van Fossen, E. M., Zhu, P., Bednar, R. M., et al. (2020). Overcoming Near-Cognate Suppression in a Release Factor 1-Deficient Host with an Improved Nitro-Tyrosine tRNA Synthetase. J. Mol. Biol. 432 (16), 4690-4704. doi:10.1016/j.jmb.2020.06.014

Cheloha, R. W., Harmand, T. J., Wijne, C., Schwartz, T. U., and Ploegh, H. L. (2020). Exploring Cellular Biochemistry with Nanobodies. J. Biol. Chem. 295 (45), 15307-15327. doi:10.1074/jbc.rev120.012960

Cigler, M., Müller, T. G., Horn-Ghetko, D., von Wrisberg, M.-K., Fottner, M., Goody, R. S., et al. (2017). Proximity-Triggered Covalent Stabilization of LowAffinity Protein Complexes In Vitro and In Vivo. Angew. Chem. Int. Ed. 56 (49), 15737-15741. doi:10.1002/anie.201706927

Clapp, C., Portt, L., Khoury, C., Sheibani, S., Norman, G., Ebner, P., et al. (2012). 14-3-3 Protects against Stress-Induced Apoptosis. Cell Death Dis 3, e348. doi: $10.1038 /$ cddis. 2012.90
Investigation: $\mathrm{EV}$ and SG. Supervision: $\mathrm{RM}$ and $\mathrm{SB}$. Writing-original draft: EV and SG. Writing-review and editing: EV, CR, DM, CC, RC, and RM.

\section{FUNDING}

This work was supported in part by grants from the National Science Foundation (Nos. NSF-1518265 and NSF-2054824), the National Institutes of Health (Nos. R01GM131168 and R01GM114653) and the Oregon State University Research and Innovation Seed Program (SciRIS) Stage 2 award and with College of Science SURE Science funds. Molecular graphics and analyses performed with UCSF Chimera, developed by the Resource for Biocomputing, Visualization, and Informatics at the University of California, San Francisco, with support from NIH P41-GM103311. Mass spectrometry was performed at the Oregon State University Mass Spectrometry Facility (OSUMSC) with NIH instrument Grant NIH \#1S10RR025628-01-Waters Ion Mobility ToF Mass Spectrometer.

\section{SUPPLEMENTARY MATERIAL}

The Supplementary Material for this article can be found online at: https://www.frontiersin.org/articles/10.3389/fchem.2022.835229/ full\#supplementary-material

Cooley, R. B., Feldman, J. L., Driggers, C. M., Bundy, T. A., Stokes, A. L., Karplus, P. A., et al. (2014a). Structural Basis of Improved Second-Generation 3-nitrotyrosine tRNA Synthetases. Biochemistry 53 (12), 1916-1924. doi:10.1021/ bi5001239

Cooley, R. B., Karplus, P. A., and Mehl, R. A. (2014b). Gleaning Unexpected Fruits from Hard-Won Synthetases: Probing Principles of Permissivity in Noncanonical Amino Acid-tRNA Synthetases. Chembiochem 15 (12), 1810-1819. doi:10.1002/cbic.201402180

Corti, C., Leclerc L'Hostis, E., Quadroni, M., Schmid, H., Durussel, I., Cox, J., et al. (1999). Tyrosine Phosphorylation Modulates the Interaction of Calmodulin with its Target Proteins. Eur. J. Biochem. 262 (3), 790-802. doi:10.1046/j.14321327.1999.00441.x

Crow, J. P., and Beckman, J. S. (1995). Quantitation of Protein Tyrosine, 3Nitrotyrosine, and 3-Aminotyrosine Utilizing HPLC and Intrinsic Ultrviolet Absorbance. Methods 7 (1), 116-120. doi:10.1006/meth.1995.1017

de Beer, M. A., and Giepmans, B. N. G. (2020). Nanobody-Based Probes for Subcellular Protein Identification and Visualization. Front. Cell. Neurosci. 14, 573278. doi:10.3389/fncel.2020.573278

DiDonato, J. A., Aulak, K., Huang, Y., Wagner, M., Gerstenecker, G., Topbas, C., et al. (2014). Site-specific Nitration of Apolipoprotein A-I at Tyrosine 166 Is Both Abundant within Human Atherosclerotic Plaque and Dysfunctional. J. Biol. Chem. 289 (15), 10276-10292. doi:10.1074/jbc. $\mathrm{m} 114.556506$

Fairhead, M., and Howarth, M. (2015). Site-specific Biotinylation of Purified Proteins Using BirA. Methods Mol. Biol. 1266, 171-184. doi:10.1007/978-14939-2272-7_12

Ferrer-Sueta, G., Campolo, N., Trujillo, M., Bartesaghi, S., Carballal, S., Romero, N., et al. (2018). Biochemistry of Peroxynitrite and Protein Tyrosine Nitration. Chem. Rev. 118 (3), 1338-1408. doi:10.1021/acs.chemrev.7b00568

Franco, M. C., Ricart, K. C., Gonzalez, A. S., Dennys, C. N., Nelson, P. A., Janes, M. S., et al. (2015). Nitration of Hsp90 on Tyrosine 33 Regulates Mitochondrial 
Metabolism. J. Biol. Chem. 290 (31), 19055-19066. doi:10.1074/jbc.m115. 663278

Franco, M. C., Ye, Y., Refakis, C. A., Feldman, J. L., Stokes, A. L., Basso, M., et al. (2013). Nitration of Hsp90 Induces Cell Death. Proc. Natl. Acad. Sci. 110 (12), E1102-E1111. doi:10.1073/pnas.1215177110

Frey, S., and Görlich, D. (2014). A New Set of Highly Efficient, Tag-Cleaving Proteases for Purifying Recombinant Proteins. J. Chromatogr. A 1337, 95-105. doi:10.1016/j.chroma.2014.02.029

Gerding, H. R., Karreman, C., Daiber, A., Delp, J., Hammler, D., Mex, M., et al. (2019). Reductive Modification of Genetically Encoded 3-nitrotyrosine Sites in Alpha Synuclein Expressed in E.Coli. Redox Biol. 26, 101251. doi:10.1016/j.redox.2019.101251

Ghesquiere, B., Colaert, N., Helsens, K., Dejager, L., Vanhaute, C., Verleysen, K., et al. (2009). In Vitro and In Vivo Protein-Bound Tyrosine Nitration Characterized by diagonal Chromatography. Mol. Cell Proteomics 8 (12), 2642-2652. doi:10.1074/mcp.m900259-mcp200

Green, M. R., and Sambrook, J. (2018). Touchdown Polymerase Chain Reaction (PCR). Cold Spring Harb Protoc. 2018 (5). doi:10.1101/pdb. prot095133

Herce-Pagliai, C., Kotecha, S., and Shuker, D. E. G. (1998). Analytical Methods for 3-nitrotyrosine as a Marker of Exposure to Reactive Nitrogen Species: a Review. Nitric Oxide 2 (5), 324-336. doi:10.1006/niox.1998.0192

Hoffmann, J. E. (2020). Bifunctional Non-canonical Amino Acids: Combining Photo-Crosslinking with Click Chemistry. Biomolecules 10 (4), 578. doi:10. 3390/biom 10040578

Ischiropoulos, H., Zhu, L., Chen, J., Tsai, M., Martin, J. C., Smith, C. D., et al. (1992). Peroxynitrite-mediated Tyrosine Nitration Catalyzed by Superoxide Dismutase. Arch. Biochem. Biophys. 298 (2), 431-437. doi:10.1016/0003-9861(92)90431-u

Jang, H. S., Gu, X., Cooley, R. B., Porter, J. J., Henson, R. L., Willi, T., et al. (2020). Efficient Site-specific Prokaryotic and Eukaryotic Incorporation of Halotyrosine Amino Acids into Proteins. ACS Chem. Biol. 15 (2), 562-574. doi:10.1021/acschembio.9b01026

Khan, M. A., Alam, K., Hassan, S. M., and Rizvi, M. M. A. (2017). Nitration of $\mathrm{H} 2 \mathrm{~B}$ Histone Elicits an Immune Response in Experimental Animals. Autoimmunity 50 (4), 232-240. doi:10.1080/08916934.2017.1347643

Leem, J., Dunbar, J., Georges, G., Shi, J., and Deane, C. M. (2016). ABodyBuilder: Automated Antibody Structure Prediction with DataDriven Accuracy Estimation. MAbs 8 (7), 1259-1268. doi:10.1080/ 19420862.2016.1205773

Mahajan, S. P., Meksiriporn, B., Waraho-Zhmayev, D., Weyant, K. B., Kocer, I., Butler, D. C., et al. (2018). Computational Affinity Maturation of Camelid Single-Domain Intrabodies against the Nonamyloid Component of AlphaSynuclein. Sci. Rep. 8 (1), 17611. doi:10.1038/s41598-018-35464-7

Moeglin, E., Desplancq, D., Stoessel, A., Massute, C., Ranniger, J., McEwen, A. G., et al. (2021). A Novel Nanobody Precisely Visualizes Phosphorylated Histone H2AX in Living Cancer Cells under Drug-Induced Replication Stress. Cancers (Basel) 13 (13), 3317. doi:10.3390/cancers13133317

Möller, M. N., Rios, N., Trujillo, M., Radi, R., Denicola, A., and Alvarez, B. (2019). Detection and Quantification of Nitric Oxide-Derived Oxidants in Biological Systems. J. Biol. Chem. 294 (40), 14776-14802. doi:10.1074/jbc. rev119.006136

Mukherjea, P., Maune, J. F., and Beckingham, K. (1996). Interlobe Communication in Multiple Calcium-Binding Site Mutants ofDrosophilacalmodulin. Protein Sci. 5 (3), 468-477. doi:10.1002/pro.5560050308

Muyldermans, S. (2013). Nanobodies: Natural Single-Domain Antibodies. Аnnu. Rev. Biochem. 82, 775-797. doi:10.1146/annurev-biochem063011-092449

Neumann, H., Hazen, J. L., Weinstein, J., Mehl, R. A., and Chin, J. W. (2008). Genetically Encoding Protein Oxidative Damage. J. Am. Chem. Soc. 130 (12), 4028-4033. doi:10.1021/ja710100d

Neumann-Staubitz, P., Lammers, M., and Neumann, H. (2021). Genetic Code Expansion Tools to Study Lysine Acylation. Adv. Biol. (Weinh) 5 (12), e2100926. doi:10.1002/adbi.202100926

Nuriel, T., Whitehouse, J., Ma, Y., Mercer, E. J., Brown, N., and Gross, S. S. (2015). ANSID: A Solid-phase Proteomic Approach for Identification and Relative Quantification of Aromatic Nitration Sites. Front. Chem. 3, 70. doi:10.3389/ fchem.2015.00070
Pacher, P., Beckman, J. S., and Liaudet, L. (2007). Nitric Oxide and Peroxynitrite in Health and Disease. Physiol. Rev. 87 (1), 315-424. doi:10.1152/physrev.00029. 2006

Pardon, E., Laeremans, T., Triest, S., Rasmussen, S. G. F., Wohlkönig, A., Ruf, A., et al. (2014). A General Protocol for the Generation of Nanobodies for Structural Biology. Nat. Protoc. 9 (3), 674-693. doi:10.1038/nprot.2014.039

Pennington, K., Chan, T., Torres, M., and Andersen, J. (2018). The Dynamic and Stress-Adaptive Signaling Hub of 14-3-3: Emerging Mechanisms of Regulation and Context-dependent Protein-Protein Interactions. Oncogene 37 (42), 5587-5604. doi:10.1038/s41388-018-0348-3

Piazza, M., Futrega, K., Spratt, D. E., Dieckmann, T., and Guillemette, J. G. (2012). Structure and of Calmodulin (CaM) Bound to Nitric Oxide Synthase Peptides: Effects of a Phosphomimetic CaM Mutation. Biochemistry 51 (17), 3651-3661. doi:10.1021/bi300327z

Pleiner, T., Bates, M., and Görlich, D. (2018). A Toolbox of Anti-mouse and Anti-rabbit IgG Secondary Nanobodies. J. Cell Biol 217 (3), 1143-1154. doi:10.1083/jcb.201709115

Porter, J. J., and Mehl, R. A. (20182018). Genetic Code Expansion: A Powerful Tool for Understanding the Physiological Consequences of Oxidative Stress Protein Modifications. Oxid Med. Cell Longev 2018, 7607463. doi:10.1155/2018/7607463

Porter, J. J., Jang, H. S., Haque, M. M., Stuehr, D. J., and Mehl, R. A. (2020). Tyrosine Nitration on Calmodulin Enhances Calcium-dependent Association and Activation of Nitric-Oxide Synthase. J. Biol. Chem. 295 (8), 2203-2211. doi:10.1074/jbc.ra119.010999

Porter, J. J., Jang, H. S., Van Fossen, E. M., Nguyen, D. P., Willi, T. S., Cooley, R. B., et al. (2019). Genetically Encoded Protein Tyrosine Nitration in Mammalian Cells. ACS Chem. Biol. 14 (6), 1328-1336. doi:10.1021/acschembio.9b00371

Radi, R. (2018). Oxygen Radicals, Nitric Oxide, and Peroxynitrite: Redox Pathways in Molecular Medicine. Proc. Natl. Acad. Sci. USA 115 (23), 5839-5848. doi:10. 1073/pnas. 1804932115

Randall, L. M., Dalla Rizza, J., Parsonage, D., Santos, J., Mehl, R. A., Lowther, W. T., et al. (2019). Unraveling the Effects of Peroxiredoxin 2 Nitration; Role of C-Terminal Tyrosine 193. Free Radic. Biol. Med. 141, 492-501. doi:10.1016/j. freeradbiomed.2019.07.016

Rogerson, D. T., Sachdeva, A., Wang, K., Haq, T., Kazlauskaite, A., Hancock, S. M., et al. (2015). Efficient Genetic Encoding of Phosphoserine and its Nonhydrolyzable Analog. Nat. Chem. Biol. 11 (7), 496-503. doi:10.1038/ nchembio. 1823

Sabir, J. S. M., Atef, A., El-Domyati, F. M., Edris, S., Hajrah, N., Alzohairy, A. M., et al. (2014). Construction of Naïve Camelids VHH Repertoire in Phage Display-Based Library. Comptes Rendus Biologies 337 (4), 244-249. doi:10. 1016/j.crvi.2014.02.004

Smallwood, H. S., Galeva, N. A., Bartlett, R. K., Urbauer, R. J. B., Williams, T. D., Urbauer, J. L., et al. (2003). Selective Nitration of Tyr99 in Calmodulin as a Marker of Cellular Conditions of Oxidative Stress. Chem. Res. Toxicol. 16 (1), 95-102. doi:10.1021/tx025566a

Smallwood, H. S., Lourette, N. M., Boschek, C. B., Bigelow, D. J., Smith, R. D., PašaTolić, L., et al. (2007). Identification of a Denitrase Activity against Calmodulin in Activated Macrophages Using High-Field Liquid Chromatography-FTICR Mass Spectrometry. Biochemistry 46 (37), 10498-10505. doi:10.1021/bi7009713

Souza, J. M., Peluffo, G., and Radi, R. (2008). Protein Tyrosine NitrationFunctional Alteration or Just a Biomarker? Free Radic. Biol. Med. 45 (4), 357-366. doi:10.1016/j.freeradbiomed.2008.04.010

Studier, F. W. (2005). Protein Production by Auto-Induction in High-Density Shaking Cultures. Protein Expr. Purif. 41 (1), 207-234. doi:10.1016/j.pep.2005.01.016

Tomin, T., Schittmayer, M., Honeder, S., Heininger, C., and Birner-Gruenberger, R. (2019). Irreversible Oxidative post-translational Modifications in Heart Disease. Expert Rev. Proteomics 16 (8), 681-693. doi:10.1080/14789450.2019. 1645602

Trier, N. H., Hansen, P. R., and Houen, G. (2012). Production and Characterization of Peptide Antibodies. Methods 56 (2), 136-144. doi:10.1016/j.ymeth.2011.12.001

Vincke, C., Gutiérrez, C., Wernery, U., Devoogdt, N., Hassanzadeh-Ghassabeh, G., and Muyldermans, S. (2012). Generation of Single Domain Antibody Fragments Derived from Camelids and Generation of Manifold Constructs. Methods Mol. Biol. 907, 145-176. doi:10.1007/978-1-61779-974-7_8

Xiang, Z., Lacey, V. K., Ren, H., Xu, J., Burban, D. J., Jennings, P. A., et al. (2014). Proximity-enabled Protein Crosslinking through Genetically Encoding 
Haloalkane Unnatural Amino Acids. Angew. Chem. Int. Ed. 53 (8), 2190-2193. doi:10.1002/anie.201308794

Xuan, W., Shao, S., and Schultz, P. G. (2017). Protein Crosslinking by Genetically Encoded Noncanonical Amino Acids with Reactive Aryl Carbamate Side Chains. Angew. Chem. Int. Ed. 56 (18), 5096-5100. doi:10.1002/anie.201611841

Zhang, H., Han, Y., Yang, Y., Lin, F., Li, K., Kong, L., et al. (2021). Covalently Engineered Nanobody Chimeras for Targeted Membrane Protein Degradation. J. Am. Chem. Soc. 143 (40), 16377-16382. doi:10.1021/jacs.1c08521

Zhang, Y., Werling, U., and Edelmann, W. (2012). SLiCE: a Novel Bacterial Cell ExtractBased DNA Cloning Method. Nucleic Acids Res. 40 (8), e55. doi:10.1093/nar/ gkr1288

Zhao, Y., Zhang, Y., Sun, H., Maroto, R., and Brasier, A. R. (2017). Selective Affinity Enrichment of Nitrotyrosine-Containing Peptides for Quantitative Analysis in Complex Samples. J. Proteome Res. 16 (8), 2983-2992. doi:10.1021/acs. jproteome.7b00275

Zhu, P., Gafken, P. R., Mehl, R. A., and Cooley, R. B. (2019). A Highly Versatile Expression System for the Production of Multiply Phosphorylated Proteins. ACS Chem. Biol. 14 (7), 1564-1572. doi:10.1021/acschembio.9b00307
Conflict of Interest: The authors declare that the research was conducted in the absence of any commercial or financial relationships that could be construed as a potential conflict of interest.

Publisher's Note: All claims expressed in this article are solely those of the authors and do not necessarily represent those of their affiliated organizations, or those of the publisher, the editors and the reviewers. Any product that may be evaluated in this article, or claim that may be made by its manufacturer, is not guaranteed or endorsed by the publisher.

Copyright $\odot 2022$ Van Fossen, Grutzius, Ruby, Mourich, Cebra, Bracha, Karplus, Cooley and Mehl. This is an open-access article distributed under the terms of the Creative Commons Attribution License (CC BY). The use, distribution or reproduction in other forums is permitted, provided the original author(s) and the copyright owner(s) are credited and that the original publication in this journal is cited, in accordance with accepted academic practice. No use, distribution or reproduction is permitted which does not comply with these terms. 